\title{
Antifungal Effects and Potential Mechanisms of Benserazide Hydrochloride Alone and in Combination with Fluconazole Against Candida albicans
}

\author{
Xueqi Chen ${ }^{1,2}$ \\ jiyong $\mathrm{Wu}^{3}$ \\ Lei $\mathrm{Sun}^{3}$ \\ Jing $\mathrm{Nie}^{3}$ \\ Shan Su ${ }^{1,2}$ \\ Shujuan Sun (D) ${ }^{1,3}$ \\ 'Department of Clinical Pharmacy, The \\ First Affiliated Hospital of Shandong First \\ Medical University \& Shandong Provincial \\ Qianfoshan Hospital, Shandong \\ Engineering and Technology Research \\ Center for Pediatric Drug Development, \\ Shandong Medicine and Health Key \\ Laboratory of Clinical Pharmacy, Jinan, \\ People's Republic of China; ${ }^{2}$ Department \\ of Pharmacy, China-Japan Friendship \\ Hospital, Beijing, People's Republic of \\ China; ${ }^{3}$ Department of Pharmacy, \\ Shandong Second Provincial General \\ Hospital, Jinan, People's Republic of China
}

Correspondence: Shujuan Sun Department of Clinical Pharmacy, The First Affiliated Hospital of Shandong First Medical University \& Shandong Provincial Qianfoshan Hospital, Shandong Engineering and Technology Research Center for Pediatric Drug Development, Shandong Medicine and Health Key Laboratory of Clinical Pharmacy, Jinan, People's Republic of China

Email sunshujuan888@I63.com
Purpose: The resistance of $C$. albicans to traditional antifungal drugs brings a great challenge to clinical treatment. To overcome the resistance, developing antifungal agent sensitizers has attracted considerable attention. This study aimed to determine the anti-Candida activity of $\mathrm{BEH}$ alone or BEH-FLC combination and to explore the underlying mechanisms.

Materials and Methods: In vitro antifungal effects were performed by broth microdilution assay and XTT reduction assay. Infected Galleria mellonella larvae model was used to determine the antifungal effects in vivo. Probes Fluo-3/AM, FITC-VAD-FMK and rhodamine $6 \mathrm{G}$ were used to study the influence of $\mathrm{BEH}$ and FLC on intracellular calcium concentration, metacaspase activity and drug efflux of $C$. albicans.

Results: BEH alone exhibited obvious antifungal activities against $C$. albicans. BEH plus FLC not only showed synergistic effects against planktonic cells and preformed biofilms within $8 \mathrm{~h}$ but also enhanced the antifungal activity in infected G. mellonella larvae. Mechanistic studies indicated that antifungal effects of drugs might be associated with the increasement of calcium concentration, activation of metacaspase activity to reduce virulence and anti-biofilms, but were not related to drug efflux.

Conclusion: BEH alone or combined with FLC displayed potent antifungal activity both in vitro and in vivo, and the underlying mechanisms were related to reduced virulence factors.

Keywords: fluconazole-resistant Candida albicans, benserazide, fluconazole, synergism, biofilm, Galleria mellonella

\section{Introduction}

The Candida genus includes many fungal pathogens, Candida albicans (C. albicans) is the main species, which can cause superficial infections and systemic infections. ${ }^{1,2}$ Candidiasis is the fourth most common nosocomial infection with relative high morbidity and approximately $50 \%$ of them were caused by C. albicans. ${ }^{3,4}$ One of the first lines of antifungal agents against $C$. albicans infections is azoles, and fluconazole (FLC) is the most commonly used agent since it is cheap, effective and low in toxicity. ${ }^{1,5}$ However, the frequent use of FLC leads to the increase in drug resistance, which has posed great challenges to clinic. Therefore, it is urgent to explore effective therapeutic strategies to combat with the drug resistance of $C$. albicans. ${ }^{6,7}$ 
Benserazide hydrochloride (BEH) is a peripheral aromatic L-amino acid decarboxylase inhibitor that can be combined with L-3,4-dihydroxyphenylalanine (L-DOPA) to treat age-related neurodegenerative disorder and Parkinson's disease. ${ }^{8}$ Currently, several studies have suggested that BEH could suppress tumor growth by inhibiting hexokinase 2 (HK2), a prominent enzyme in glycolysis pathway. ${ }^{9}$ HK2 has been proved to be necessary for the hyphal growth and full virulence of C. albicans. ${ }^{10,11}$ Furthermore, the combination of BEH and L-DOPA has an inhibitory effect on Staphylococcus aureus with MIC of $1.25 \mathrm{mg} / \mathrm{mL}$ and $5 \mathrm{mg} / \mathrm{mL}$, respectively, but the drug combination exhibits no effects on C. albicans. ${ }^{12}$ However, no reports have illustrated the antifungal activity of BEH alone or combined with FLC against C. albicans.

In the present study, the antifungal activities of BEH alone or combined with FLC against $C$. albicans planktonic cells were evaluated by the chequerboard microdilution method, and the XTT reduction assay was conducted to determine the antibiofilm effects. In addition, the antifungal effects of the drug combination against $C$. albicans in vivo were evaluated by establishing a Galleria mellonella (G. mellonella) larva infection model and measuring the number of surviving larvae, fungal cells burden, and the degree of tissue damage. Furthermore, potential antifungal mechanisms were explored by assessing intracellular calcium fluctuations, the activation of metacaspase and drug efflux in resistant C. albicans.

\section{Materials and Methods}

\section{Isolates and Growth Media}

The isolates used in this study are listed in Table 1, including six FLC-resistant $C$. albicans and six FLC-susceptible C. albicans. CA103, CA632, and CA20003 were kindly provided by Professor Changzhong Wang (School of Integrated Traditional and Western Medicine, Anhui University of Traditional Chinese Medicine, Hefei, China), and the rest of strains were isolated from the clinical laboratory of Shandong Provincial Qianfoshan Hospital, Jinan, China. The quality control strain C. albicans ATCC 10231 was kindly provided by the Institute of Pharmacology, School of Pharmacy, Shandong University, Jinan, Shandong Province, China. All strains were stored at $-80^{\circ} \mathrm{C}$ and were subcultured at least three times on Sabouraud dextrose agar (SDA) solid medium.

\section{Drugs and $G$. mellonella Larvae}

Drugs and G. mellonella larvae were bought from Dalian Meilun Biotech Co., Ltd. (Dalian, China) and the Biotech Co., Ltd. of Tianjin Huiyude (Tianjin, China), respectively. The stock solutions of FLC and BEH were prepared to $2560 \mu \mathrm{g} / \mathrm{mL}$ and $1024 \mu \mathrm{g} / \mathrm{mL}$ in sterile distilled water. We selected larvae of appropriate age with moderate body

Table I Drug Interactions of BEH Used Alone or in Combination with FLC Against Candida albicans

\begin{tabular}{|c|c|c|c|c|c|c|}
\hline \multirow[t]{3}{*}{ Isolates } & \multicolumn{4}{|c|}{ MIC $(\mu g / m L)$} & \multirow[t]{3}{*}{$\mathbf{F I C l}$} & \multirow[t]{3}{*}{ Interpretation } \\
\hline & \multicolumn{2}{|c|}{ Alone } & \multicolumn{2}{|c|}{ Combined } & & \\
\hline & FLC & BEH & FLC & BEH & & \\
\hline CAIO(R) & $>512$ & 32 & 0.125 & 8 & 0.25 & SYN \\
\hline CAI6(R) & $>512$ & 32 & 0.25 & 8 & 0.25 & SYN \\
\hline CAI03(R) & $>512$ & 16 & 0.25 & 4 & 0.25 & SYN \\
\hline CAI37(R) & $>512$ & 32 & 0.5 & 4 & 0.125 & SYN \\
\hline CA632(R) & $>512$ & 32 & 0.5 & 8 & 0.25 & SYN \\
\hline CA20003(R) & $>512$ & 16 & 0.125 & 8 & 0.5 & SYN \\
\hline CA4(S) & 0.5 & 16 & 0.25 & 2 & 0.625 & $\mathrm{NI}$ \\
\hline CA8(S) & 0.5 & 8 & 0.25 & 2 & 0.75 & $\mathrm{NI}$ \\
\hline $\mathrm{CAI} 4(\mathrm{~S})$ & 0.5 & 16 & 0.25 & I & 0.562 & $\mathrm{NI}$ \\
\hline CA20(S) & 0.125 & 32 & 0.125 & 8 & 1.25 & $\mathrm{NI}$ \\
\hline CAI9(S) & 0.125 & 32 & 0.125 & 8 & 1.25 & $\mathrm{NI}$ \\
\hline CAI29(S) & I & 32 & 0.5 & 4 & 0.75 & $\mathrm{NI}$ \\
\hline
\end{tabular}

Notes: MIC denotes minimum inhibitory concentration of drug that inhibited fungal growth by $80 \%$ compared with the control group. MIC values and FICls are shown as the median of three independent experiments.

Abbreviations: CA, Candida albicans; BEH, benserazide hydrochloride; FLC, fluconazole; $\mathrm{FICl}$, fractional inhibitory concentration index; SYN, synergism; NI, no interaction. 
length and weights, and then divided them into groups randomly.

\section{Antifungal Effects of BEH and FLC Against C. albicans in vitro Determination of Minimal Inhibitory Concentrations (MICs)}

This experiment followed the M27-A3 document ${ }^{13}$ issued by the Clinical and Laboratory Standards Institute (CLSI) with some modifications. We used the microbroth dilution method to determine the antifungal effects of BEH and FLC on 96-well plates against C. albicans. ${ }^{14,15}$ Each experiment was conducted once every $24 \mathrm{~h}$, repeated at least 3 times, and finally, we recorded the median drug concentrations. Specifically, we added $100 \mu \mathrm{L}$ fungal suspension $\left(2 \times 10^{3} \mathrm{CFU} / \mathrm{mL}\right), 50 \mu \mathrm{L} \mathrm{BEH}(4-64 \mu \mathrm{g} / \mathrm{mL})$ and FLC $(0.125-64 \mu \mathrm{g} / \mathrm{mL})$ to $96-w e l l$ plates. The wells in column 12 containing $200 \mu \mathrm{L}$ RPMI-1640 were set as control groups, and the drug-free well was served as the growth control group. Then, the 96-well plate was placed for 24 hours. The growth inhibition was observed by eyes, and the optical density (OD) was measured by a microplate reader. The MIC endpoint was defined as the lowest concentration of drug that reduced growth by $80 \%$ compared to the growth control group. ${ }^{6,16}$ The synergistic effects on planktonic cells in vitro were interpreted by the fractional inhibitory concentration index (FICI) model using the following calculating equation: ${ }^{16} \mathrm{FICI}=$ $\mathrm{FIC}_{B E H}+\mathrm{FIC}_{F L C}=\left(\mathrm{MIC}_{80}\right.$ of $\mathrm{BEH}$ in combination $/ \mathrm{MIC}_{80}$ of $\mathrm{BEH}$ alone $)+\left(\mathrm{MIC}_{80}\right.$ of $\mathrm{FLC}$ in combination/ $\mathrm{MIC}_{80}$ of FLC alone). In this model, we regarded the combination of drugs as a synergistic effect when the FICI value was $\leq 0.5$; the combination of drugs has an antagonistic effect when the FICI value was $>4$; and when $0.5<$ FICI $<4$, the combination of the two drugs showed an irrelevant effect. ${ }^{17}$

\section{Determination of Sessile Minimum Inhibitory Concentrations (sMICs) of Biofilms}

The sMIC values of FLC combined with BEH against C. albicans biofilms were measured at 96-well microtiter plates at different times as previously described, with slight changes. ${ }^{18,19}$ Similarly, each experiment was conducted once every $24 \mathrm{~h}$, repeated at least 3 times, and finally, we recorded the median drug concentrations. Specifically, C. albicans biofilms at four different time points $(4,8,12$, and 24 hours) were formed by adding yeast suspensions $\left(2 \times 10^{3} \mathrm{CFU} / \mathrm{mL}\right)$ into each well. Then, at the above time points, we washed each well for three times to remove the planktonic yeast cells. Moreover, $100 \mu \mathrm{L}$ BEH dilutions $(16-1024 \mu \mathrm{g} / \mathrm{mL})$ and FLC dilutions $(2-1024 \mu \mathrm{g} / \mathrm{mL})$ were added to the wells except for the drug-free group. Subsequently, we used XTT [2,3-bis(2-methoxy-4-nitro-5-sulfophenyl)- $2 \mathrm{H}$ - tetrazolium-5-carboxanilide] reduction assay to evaluate the anti-biofilm activity of drugs. We used a microplate reader to measure the colorimetric change of XTT reduction at $492 \mathrm{~nm}$ and recorded the results. In this experiment, FICI model was used to interpret the interaction between BEH and FLC against $C$. albicans biofilms. sMIC value was regarded as the lowest concentration at which $80 \%$ of the biofilm metabolic activity was inhibited.

\section{Antifungal Effects of BEH and FLC Against Candida albicans in vivo G. mellonella Larvae Survival Assay}

The larvae survival assay was performed according to the previously described methodology with some modifications. ${ }^{16,20}$ Before the experiment, 80 larvae were randomly divided into the following groups: control group, FLC group, BEH group, and BEH-FLC combination group. At first, each larva was injected with $10 \mu \mathrm{L}$ fungal suspension $\left(5 \times 10^{8} \mathrm{CFU} / \mathrm{mL}\right)$ through the last right proleg. After 2 hours of infection, each larva was injected with $10 \mu \mathrm{L}$ phosphate buffer solution (PBS), FLC (160 $\mu \mathrm{g} /$ $\mathrm{mL})$, BEH $(96 \mu \mathrm{g} / \mathrm{mL})$ or FLC + BEH through the last left proleg, respectively. Then, they were cultured at $35^{\circ} \mathrm{C}$ and recorded the number of surviving larvae in each group every 24 hours. We considered they were dead when larvae became black or soft and there was no obvious tactile response. The recording lasted for 4 days, and the experiment was repeated at least 3 times. Finally, SPSS software was used for survival analysis (Kaplan-Meier method), and survival curves of different groups of larvae were drawn.

\section{Fungal Burden Determination}

First, we constructed larvae-C. albicans infection model in accordance with the method described above. Then we randomly selected 3 larvae from each group every 24 hours (regardless of whether they were dead or alive), then placed them in $3 \mathrm{~mL}$ sterile PBS solution, and grinded them with a high-speed homogenizer. The homogenate was diluted for multiple times and inoculated on the Yeast Extract Peptone Dextrose (YPD) medium. Subsequently, we drew $10 \mu \mathrm{L}$ of homogenates under 
different concentration gradients and spotted them on sterile YPD medium. After the colonies grow on the culture medium, the numbers of fungal cells were recorded by the colony counting method. ${ }^{21,22}$ We then converted it to obtain the $C$. albicans content of each larva in different groups before dilution. Finally, we used the unpaired $t$-test pair to analyze the results.

\section{Histological Study}

We firstly constructed larvae-C. albicans infection model in accordance with the method described above. After post-infection for 2 days, we randomly selected 5 larvae from each group, and cut them into sections with a thickness of about $18-22 \mu \mathrm{m}$. Subsequently, these tissue sections were stained with a regular periodic acid-Schiff (PAS) staining step and dehydrated in different ethanol solutions. Then, we observed and photographed tissue sections under the microscope with $4.2 \times$ magnification (Leica DMi 8, Germany).

\section{Detection of BEH on Cytosolic Calcium Concentration $\left(\left[\mathrm{Ca}^{2+}\right]_{i}\right)$}

The measurement of $\left[\mathrm{Ca}^{2+}\right]_{\mathrm{i}}$ fluctuations in C. albicans (CA 10) was performed by using fluorescent probe Fluo3/AM (DMSO, Sigma-Aldrich, MO, USA). The experiment was conducted as previously described. ${ }^{22,23}$ Briefly, CA10 yeast cells were activated by YPD medium, and then they were harvested and washed three-times by D hank's buffer solution (calcium ion, magnesium ion and phenol red free, Solarbio, Beijing, China). Moreover, yeast cells were re-suspended to the final concentration of $10^{7} \mathrm{CFU} / \mathrm{mL}$ and were stained with $5 \mu \mathrm{M}$ Fluo-3/AM for $45 \mathrm{~min}$ at $37^{\circ} \mathrm{C}$ in the dark. Then, solutions containing FLC $(1 \mu \mathrm{g} / \mathrm{mL}), \mathrm{BEH} / \mathrm{FLC}(8 \mu \mathrm{g} / \mathrm{mL}+1 \mu \mathrm{g} / \mathrm{mL}$, respectively), BEH ( $2 \mu \mathrm{g} / \mathrm{mL}, 4 \mu \mathrm{g} / \mathrm{mL}, 8 \mu \mathrm{g} / \mathrm{mL}$, respectively) was added to the experimental groups, and the control group that was only treated with above Fluo-3/ AM. $\left[\mathrm{Ca}^{2+}\right]_{\mathrm{i}}$ was calculated as follows: ${ }^{24}$

$$
\left[\mathrm{Ca}^{2+}\right]_{\mathrm{i}}=\mathrm{K}_{\mathrm{d}} \times\left[\left(\mathrm{F}-\mathrm{F}_{\min }\right) /\left(\mathrm{F}_{\max }-\mathrm{F}\right)\right]
$$

In this formula, $\mathrm{K}_{\mathrm{d}}$ is the effective dissociation constant of calcium binding to Fluo-3/AM $\left(204 \mathrm{nmol} / \mathrm{l}\right.$ at $\left.37^{\circ} \mathrm{C}\right)$. $\mathrm{F}_{\text {min }}$ is the background fluorescence intensity with $0.1 \%$ Triton $\mathrm{X}-100$ and $0.1 \mathrm{mM}$ EDTA solution, while $\mathrm{F}_{\max }$ is the maximum fluorescence intensity with $0.1 \%$ Triton $\mathrm{X}-100$ plus $1 \mathrm{mM} \mathrm{CaCl} 2$ solution. $\mathrm{F}$ is the mean fluorescence intensity (MFI) for each sample. $\left[\mathrm{Ca}^{2+}\right]_{\mathrm{i}}$ was determined by a flow cytometer (Becton Dickinson FACSARIAII,
United States) at an excitation wavelength of $488 \mathrm{~nm}$ and an emission wavelength of $525 \mathrm{~nm}$. Fluctuations of $\left[\mathrm{Ca}^{2+}\right]_{\mathrm{i}}$ were detected every $10 \mathrm{~min}$ for $50 \mathrm{~min}$. In this experiment, we selected a series of drug concentrations based on MIC and different fungal suspension concentrations, and finally selected the optimal drug concentrations.

\section{Detection of Metacaspase Activity}

In this experiment, the reagent FITC-VAD-FMK In Situ Marker (Promega, Madison, WI, United States) was used to explore whether the antifungal effects of BEH-FLC against drug-resistant $C$. albicans were related to the activation of intracellular metacaspase. ${ }^{6,25}$ In detail, C. albicans suspension $\left(5 \times 10^{6} \mathrm{CFU} / \mathrm{mL}\right)$ was treated with FLC $(1 \mu \mathrm{g} / \mathrm{mL})$, BEH $(32 \mu \mathrm{g} / \mathrm{mL})$, and BEH plus FLC for $16 \mathrm{~h}$, respectively. After centrifugation, yeast cells were incubated with FITC-VAD-FMK probes for 1 h. Subsequently, we collected cell suspension from each group to glass slides, then observed and photographed the fluorescence of fungal cells using the microscope (Leica DMi 8, Germany). In this experiment, we selected a series of drug concentrations based on MIC and different fungal suspension concentrations, and finally selected the optimal concentrations: $1 \mu \mathrm{g} / \mathrm{mL}$ and $32 \mu \mathrm{g} / \mathrm{mL}$.

\section{FLC Efflux Assay}

In order to clarify the effect of BEH on drug transporters, the fluorescent Rh6G was used as a substitute for FLC to determine whether BEH has an effect on FLC efflux. ${ }^{22,26}$ After incubating, yeast cells $\left(1 \times 10^{5} \mathrm{CFU} / \mathrm{mL}\right)$ were centrifuged and washed with PBS solutions for twice. Afterwards, the fungal cells were shaken for $1 \mathrm{~h}$ to completely deplete the ATP. Subsequently, we added Rh6G solution $(10 \mu \mathrm{M})$ to the cell suspension for $70 \mathrm{~min}$ of incubation and re-suspended in $5 \mathrm{~mL}$ Glu-PBS (5\% glucose). Meanwhile, Rh6G-alone group was the control group, and an appropriate amount of BEH was added to make the final working concentration at $32 \mu \mathrm{g} / \mathrm{mL}$. The detection conditions of the flow cytometer (Becton Dickinson FACSARIA-II, USA) were excitation wavelength $488 \mathrm{~nm}$ and emission wavelength $530 \mathrm{~nm}$. We measured every 30 minutes for a total of 150 minutes, and recorded the data. In this experiment, we selected a series of drug concentrations based on MIC and different fungal suspension concentrations, and finally selected BEH optimal concentration: $32 \mu \mathrm{g} / \mathrm{mL}$. 


\section{Statistics}

All experiments were conducted at least three times independently. Graphs and statistical analyses were performed with GraphPad Prism $7^{27}$ (version 7.3.0, GraphPad, La Jolla, CA, www.graphpad.com) and IBM SPSS Statistics $22^{28}$ (version 22, SPSS, Chicago, IL, https:/www.ibm.com/products/spss-statistics). Survival assay was analyzed by Kaplan-Meier method, and fungal burden determination and FLC efflux assay were analyzed using an unpaired $t$-test. $P<0.05$ was considered significant.

\section{Results}

\section{Antifungal Effects of $\mathrm{BEH}$ and FLC Against} C. albicans in vitro

MICs of BEH Alone and in Combination with FLC Against $C$. albicans

The MIC values of BEH and FLC against 12 C. albicans are summarized in Table 1. It can be seen that BEH alone had obvious antifungal activity against FLC-resistant and FLC-sensitive C. albicans, MICs of BEH were 16-32 $\mu \mathrm{g}$ / $\mathrm{mL}$ and $8-32 \mu \mathrm{g} / \mathrm{mL}$, respectively. It also showed that BEH plus FLC had synergistic effects against resistant
C. albicans with FICIs $0.125-0.5$, which led to a decrease in the MICs of BEH from $16-32 \mu \mathrm{g} / \mathrm{mL}$ to $4-$ $8 \mu \mathrm{g} / \mathrm{mL}$ and the MICs of FLC from $>512 \mu \mathrm{g} / \mathrm{mL}$ to $0.125-0.5 \mu \mathrm{g} / \mathrm{mL}$. Although no synergism was observed when BEH was combined with FLC against FLCsusceptible $C$. albicans, the MICs of BEH could be reduced by $4-16$ times.

\section{sMICs of BEH Combined with FLC Against Biofilms of $C$. albicans}

Table 2 summarizes the effect of BEH plus FLC against the preformed biofilms of $4 \mathrm{C}$. albicans isolates. The results exhibited that the combination of BEH and FLC had a synergistic effect on the biofilm formed within 8 hours but showed no synergistic activity on the biofilm formed over 12 hours. BEH plus FLC showed a strong synergistic effect against the biofilm formed on $4 \mathrm{~h}$, and the sMIC value of FLC could be reduced from $>1024 \mu \mathrm{g}$ / $\mathrm{mL}$ to $0.5-2 \mu \mathrm{g} / \mathrm{mL}$. In addition, for the biofilm preformed at $8 \mathrm{~h}$, FICIs $<0.5$, and the sMIC values of BEH and FLC were decreased from $>1024 \mu \mathrm{g} / \mathrm{mL}$ to $16 \mu \mathrm{g} / \mathrm{mL}$ and $4 \mu \mathrm{g} /$ $\mathrm{mL}$, respectively. However, no synergism was observed for biofilms that were preformed for more than 12 hours, and the FICI values $>0.5$.

Table 2 In vitro Interactions of BEH with FLC Against Biofilms of Candida albicans

\begin{tabular}{|c|c|c|c|c|c|c|c|}
\hline \multirow[t]{3}{*}{ Isolate } & \multirow[t]{3}{*}{ Time $(h)^{a}$} & \multicolumn{4}{|c|}{ sMIC $(\mu \mathrm{g} / \mathrm{mL})$} & \multirow[t]{3}{*}{$\mathbf{F I C l}$} & \multirow[t]{3}{*}{ Interpretation } \\
\hline & & \multicolumn{2}{|c|}{ Alone } & \multicolumn{2}{|c|}{ Combined } & & \\
\hline & & FLC & BEH & FLC & BEH & & \\
\hline \multirow[t]{4}{*}{ CAIO(R) } & 4 & $>1024$ & 32 & 2 & 8 & 0.252 & SYN \\
\hline & 8 & $>1024$ & 1024 & 4 & 16 & 0.02 & SYN \\
\hline & 12 & $>1024$ & $>1024$ & 64 & 512 & 0.625 & $\mathrm{NI}$ \\
\hline & 24 & $>1024$ & $>1024$ & 64 & 512 & 0.625 & $\mathrm{NI}$ \\
\hline \multirow[t]{4}{*}{ CAI6(R) } & 4 & $>1024$ & 32 & 2 & 8 & 0.252 & SYN \\
\hline & 8 & $>1024$ & 1024 & 4 & 16 & 0.02 & SYN \\
\hline & 12 & $>1024$ & $>1024$ & 64 & 512 & 0.625 & $\mathrm{NI}$ \\
\hline & 24 & $>1024$ & $>1024$ & 64 & 512 & 0.625 & $\mathrm{NI}$ \\
\hline \multirow[t]{4}{*}{ CA4(S) } & 4 & $>1024$ & 16 & I & 4 & $0.25 \mathrm{I}$ & SYN \\
\hline & 8 & $>1024$ & $>1024$ & 4 & 16 & 0.2 & SYN \\
\hline & 12 & $>1024$ & $>1024$ & 512 & 512 & I & $\mathrm{NI}$ \\
\hline & 24 & $>1024$ & $>1024$ & 512 & 512 & I & $\mathrm{NI}$ \\
\hline \multirow[t]{4}{*}{ CA8(S) } & 4 & $>1024$ & 16 & 0.5 & 4 & 0.250 & SYN \\
\hline & 8 & $>1024$ & $>1024$ & 4 & 16 & 0.252 & SYN \\
\hline & 12 & $>1024$ & $>1024$ & 1024 & 512 & 1.5 & $\mathrm{NI}$ \\
\hline & 24 & $>1024$ & $>1024$ & 1024 & 512 & 1.5 & $\mathrm{NI}$ \\
\hline
\end{tabular}

Notes: sMIC denotes the sessile minimum inhibitory concentration of drug that produced an $80 \%$ reduction of biofilms metabolic activity compared with the growth control. sMIC values and $\mathrm{FICls}$ are shown as the median of three independent experiments. ${ }^{\mathrm{a}}$ Time indicates the incubation period of preformed biofilms.

Abbreviations: CA, Candida albicans; benserazide hydrochloride; FLC, fluconazole; $\mathrm{FICl}$, fractional inhibitory concentration index; SYN, synergism; NI, no interaction. 


\section{G. mellonella Larvae Infection Model G. mellonella Survival Assay}

The survival numbers of larvae infected by $C$. albicans and treated with drugs within 4 days was significantly different as shown in Figure 1A. The results showed that the survival rates after 4 days of BEH group (40\%) and FLC group (35\%) had a slight increase compared with the control group $(P>0.5)$, while the BEH-FLC combination group could increase the survival rates $(85 \%)$ significantly $(P<0.01)$. The difference in survival rates indicated that BEH plus FLC could obviously prolong the survival time and improve survival rates of G. mellonella larvae compared with other groups.

\section{Fungal Burden Determination}

The number of fungi in larvae infected by $C$. albicans and treated with drugs within 4 days was also different, as illustrated in Figure 1B. During the 4-day period, the content of fungi in all groups increased gradually over time. Treatment with FLC or BEH alone only led to a slight decrease on C. albicans numbers in larvae compared with the control group. However, FLC combined with BEH resulted in a significant decrease in fungi number compared to other groups $(P<0.01)$. In addition, the data of BEH alone group are not shown in Figure 1B since it is very similar to FLC group.

\section{Histological Study}

As illustrated in Figure 2, the degree of tissue damage to larvae treated with different drugs was different. There were a large number of black clumps and tissue infiltration areas in the control group. The areas and numbers of fungal clumps in BEH single group and FLC single group were reduced slightly, while in BEH-FLC combination group, the clumps and the number were significantly reduced, and only small dot-like clumps were scattered in larvae tissue. The observation of the histological study was consistent with the fungal burden determination.

\section{Detection of $\mathrm{BEH}$ on Fluctuations of $\left[\mathrm{Ca}^{2+}\right]_{\mathrm{i}}$}

The fluctuation of intracellular calcium is a potential antifungal mechanism for many antifungal compounds. The results showed that, after 20 minutes, $8 \mu \mathrm{g} / \mathrm{mL}$ BEH could significantly increase the intracellular calcium ion concentration compared with the control group $(P<0.001$, Figure 3A). For the drug combination experiment, after 20 minutes, we could see that both the BEH single-use group and the combination group can increase the calcium concentration, but there was no significant difference between the two curves (Figure 3B). Therefore, the increase of intracellular $\left[\mathrm{Ca}^{2+}\right]_{\mathrm{I}}$ concentrations may be one of the potential antifungal mechanisms for BEH alone against $C$. albicans (CA10).

\section{Metacaspase Activation}

The results showed that, compared with control group, neither FLC alone group nor BEH alone group produced green fluorescence under the microscope. We observed that the number of fungal cells was reduced both in BEH alone group and BEH-FLC combination group, but the bright green fluorescence was produced only in BEHFLC group (Figure 4), which indicated that the metacaspase in $C$. albicans was activated by BEH plus FLC.
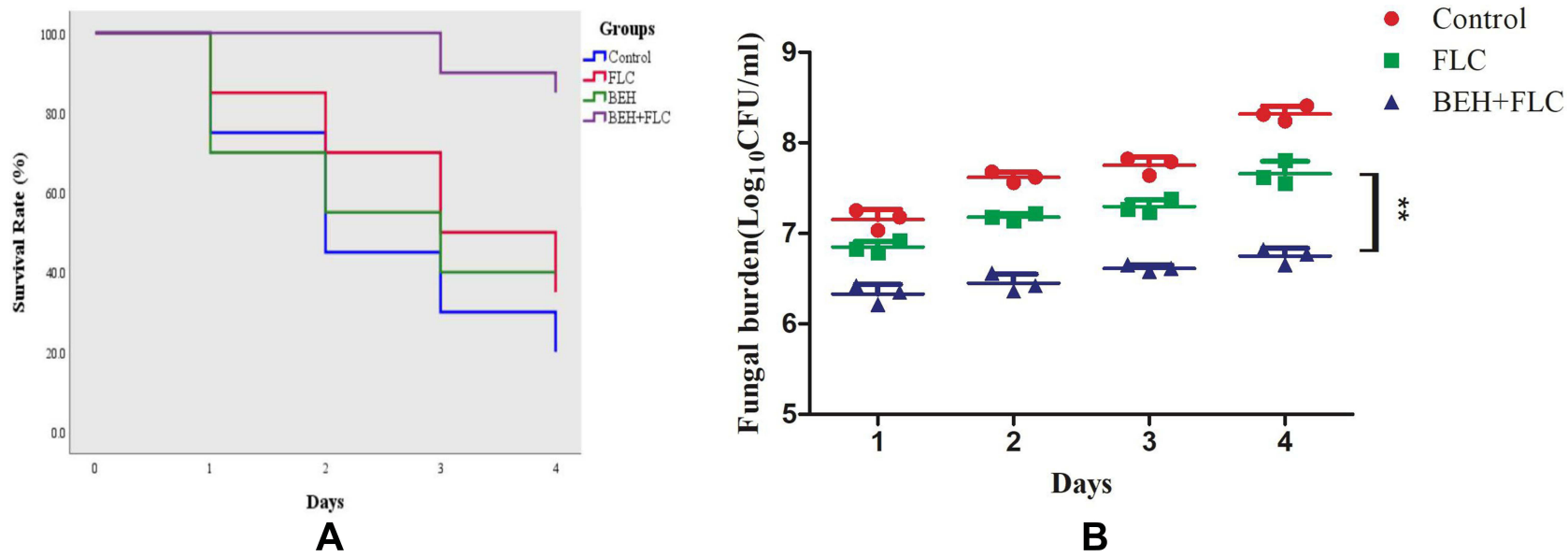

Figure I Survival curve (A) and Fungal burden (B) of Galleria mellonella infected with Candida albicans. The concentration of yeast cells was $5 \times 10^{8} \mathrm{CFU} /$ larva. Treatments consisted of phosphate buffered saline (PBS), fluconazole (FLC) $(160 \mu \mathrm{g} / \mathrm{mL})$, benserazide hydrochloride (BEH) $(96 \mu \mathrm{g} / \mathrm{mL})$, and a combination of FLC ( $160 \mu \mathrm{g} / \mathrm{mL}) \mathrm{with} \mathrm{BEH}$ $(96 \mu \mathrm{g} / \mathrm{mL})$. For clarity, data for treatment with BEH (B) are not shown because the data obtained closely followed those for the control group. Data are the median of three independent experiments. 


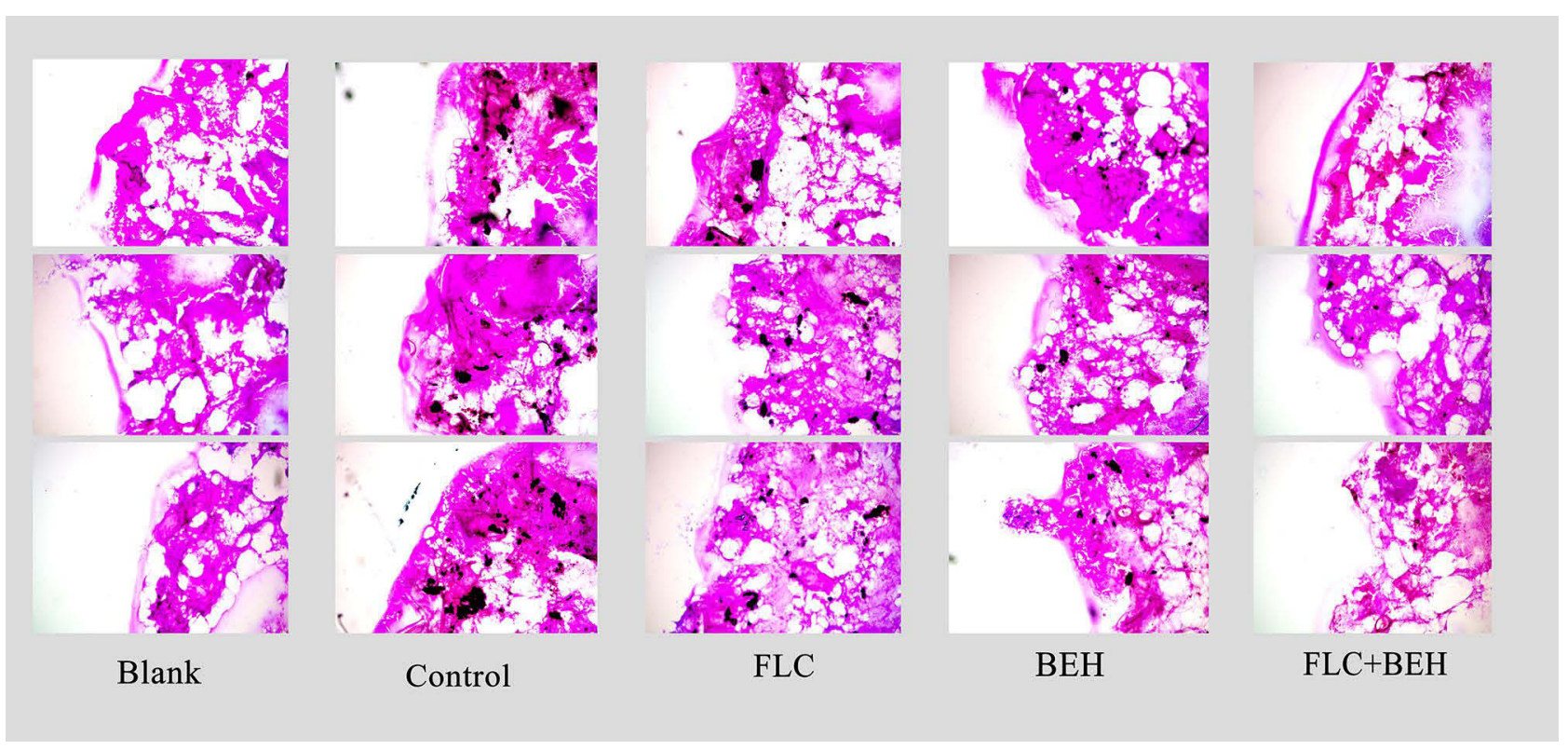

Figure 2 Histopathology of Galleria mellonella infected with Candida albicans. The concentration of yeast cells was $5 \times 10^{8} \mathrm{CFU} /$ larva. Treatments consisted of phosphate buffered saline (PBS), fluconazole (FLC) $(160 \mu \mathrm{g} / \mathrm{mL})$, benserazide hydrochloride $(\mathrm{BEH})(96 \mu \mathrm{g} / \mathrm{mL})$, and a combination of FLC and BEH. Melanized nodules were the mixtures of yeast cells and filaments. The photographs were from three independent experiments.
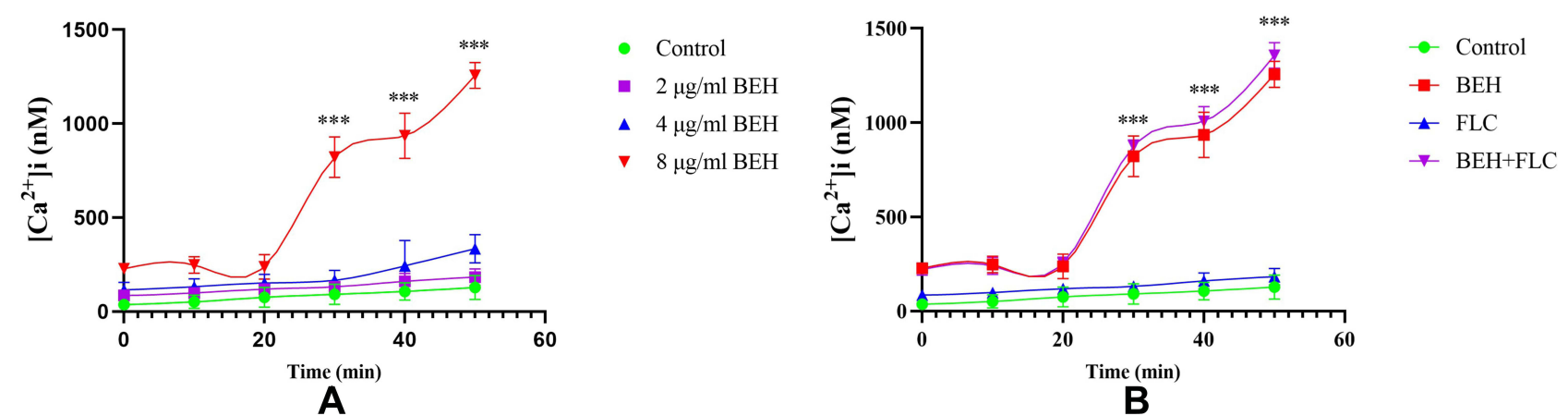

Figure 3 Detection of benserazide hydrochloride $(\mathrm{BEH})$ alone $(\mathbf{A})$ and in combination with fluconazole (FLC) (B) on Fluctuations of [Ca $\left.{ }^{2+}\right]_{i}$ in $C$. albicans. Yeast concentrations were adjusted to $10^{7} \mathrm{CFU} / \mathrm{mL}$. (A) The concentrations of $\mathrm{BEH}$ were 2,4 , and $8 \mu \mathrm{g} / \mathrm{mL}$, respectively. (B) The concentrations of BEH were $8 \mu \mathrm{g} / \mathrm{mL}$ when combined with I $\mu \mathrm{g} / \mathrm{mL}$ FLC. Data are the median of three independent experiments. $* * * P<0.00 \mathrm{I}$.

Therefore, the activation of metacaspase in FLC-resistant C. albicans may be one of the potential mechanisms for BEH plus FLC to show a synergistic antifungal effect.

\section{FLC Efflux Assay}

Since both reagent-rhodamine $6 \mathrm{G}(\mathrm{Rh} 6 \mathrm{G})$ and FLC were the substrates of Cdr1p and Cdr2p in drug efflux transporters, Rh6G was often used as a fluorescent tracer to replace FLC for monitoring the effect of drugs on drug transporters in C. albicans. ${ }^{29}$ In the drug efflux experiment, the results are shown in Figure 5. The concentration of Rh6G continued to decrease gradually both in BEH group and the control group over time, and the curves of two groups were highly similar $(P>0.05)$, which suggested that $\mathrm{BEH}$ did not inhibit intracellular FLC efflux. Therefore, the synergistic antifungal effects of $\mathrm{BEH}$ in combination with FLC against resistant $C$. albicans were independent of inhibiting FLC efflux.

\section{Discussion}

At present, the increasing number of multidrug resistant (MDR) pathogen strains has been regarded as one of the most serious public health problems in the world, posing challenges to the global health system. ${ }^{30}$ In order to deal with this problem, many studies have been carried out on the discovery of antibacterial sensitizers or new antibacterial compounds. Researchers have identified that a variety of plant essential oils have obvious antifungal activities against 

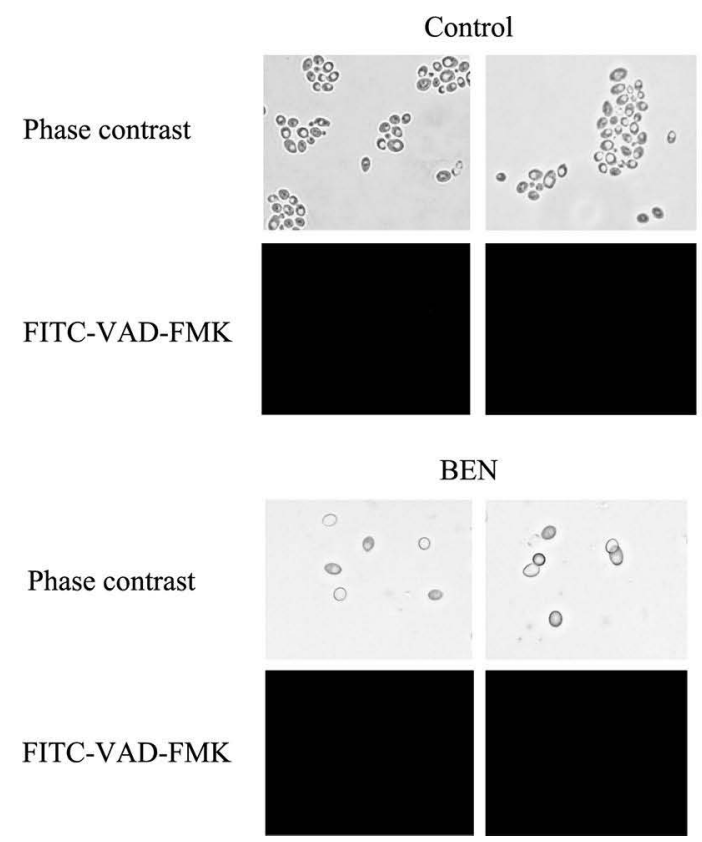

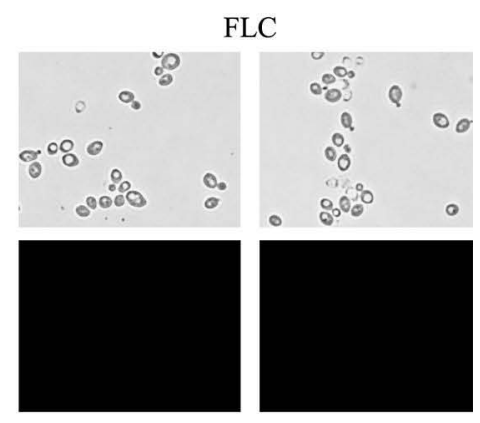

BEN+FLC

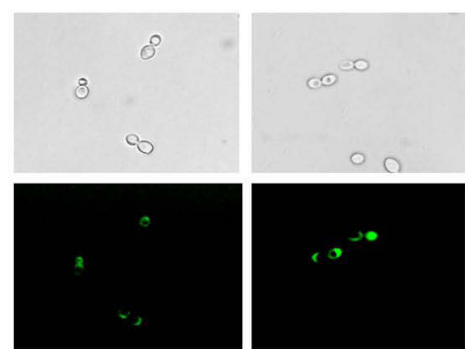

Figure 4 Effect of benserazide hydrochloride $(\mathrm{BEH})$ alone or in combination with fluconazole (FLC) on metacaspase activity. CAI0 cells were collected, stained with FITCVAD-FMK, and observed under a fluorescent microscope after treating with FLC (I $\mu \mathrm{g} / \mathrm{mL}), \mathrm{BEH}(32 \mu \mathrm{g} / \mathrm{mL})$, and a combination of FLC (I $\mu \mathrm{g} / \mathrm{mL})$ with BEH ( $32 \mu \mathrm{g} / \mathrm{mL})$. The photographs were collected from three independent experiments.

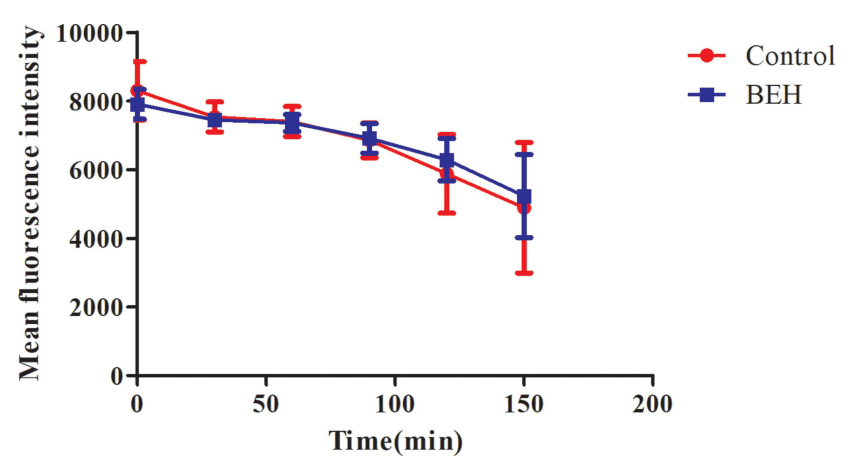

Figure 5 Effect of benserazide hydrochloride $(B E H)$ on the efflux of Rh6G in C. albicans. The efflux of Rh6G in the absence and presence of BEH $(32 \mu \mathrm{g} / \mathrm{mL})$ were determined by a flow cytometer. Mean fluorescence intensity represents the intracellular Rh6G in C. albicans. Data are the median of three independent experiments.

different Candida species. $^{31,32}$ Several studies have shown that the combination of plant essential oils and traditional antifungals could greatly reduce MDR incidence. ${ }^{32}$ In addition, commercial ophthalmic solutions containing PVP-I $0.6 \%$ or $0.05 \%$ Hexamidine Diisethionate both showed obvious antibacterial activity against MDR strains in vitro. ${ }^{33,34}$ Furthermore, several non-antifungal drugs were proved to be able to reduce the MIC value of FLC and increase its sensitivity to C. albicans, such as: ambroxol hydrochloride, budesonide, ribavirin and N-Butylphthalide. $7,16,35,36$

In the present study, we found that $\mathrm{BEH}$, which is often combined with L-DOPA for the treatment of Parkinson's disease clinically, exerts potent antifungal effects when used alone or in combination with FLC against C. albicans. It can be seen that $\mathrm{MIC}$ values of $\mathrm{BEH}$ alone were $8-32 \mu \mathrm{g} / \mathrm{mL}$ for FLC-susceptible $C$. albicans and $16-32 \mu \mathrm{g} / \mathrm{mL}$ for FLC-resistant strains in Table 1. It was also showed that BEH plus FLC had synergistic effects when, against FLC resistant $C$. albicans with FICIs ranging from 0.125 to 0.5 , the MICs of $\mathrm{BEH}$ and FLC could be reduced by 4 times and 1024-4096 times, respectively. Although no synergism was observed when BEH plus FLC against FLC-susceptible strains, the MICs of BEH could be obviously reduced.

Biofilm formation of $C$. albicans carries negative impacts on the treatment of fungal infections, since it increases the resistance to antifungal agents and the ability against host immune defenses. ${ }^{3,37}$ Moreover, the formation of $C$. albicans biofilm on medical devices can not only lead to the failure of the clinical treatment but also become the source for continued infections in the future. ${ }^{3,37}$ In this study, we found that BEH plus FLC showed a strong synergistic effect against the biofilm formed on $4 \mathrm{~h}$ and 8 $\mathrm{h}$, FICIs $<0.5$. The sMICs of FLC were reduced from $>1,024 \mu \mathrm{g} / \mathrm{mL}$ to $0.5-2 \mu \mathrm{g} / \mathrm{mL}$ and $4 \mu \mathrm{g} / \mathrm{mL}$, respectively, and the sMICs of BEH can be reduced by 4 times and 64 times, respectively. No synergism was observed for biofilms that were preformed for more than 12 hours. These 
findings indicated that the biofilm would become more mature and more complex over time, and the drug resistance would increase. Therefore, BEH-FLC drug combination may provide potential application value for early treatment of biofilm-related infections, but the effect on mature biofilms was limited.

$\mathrm{BEH}$ in combination with FLC not only exhibited synergistic effects in vitro but also enhanced the antifungal activity in vivo. In the present study, G. mellonella larvae model was used to perform the in vivo experiments. Compared with mammalian models, the larvae models have a lot of advantages in economy, ethics and operation. ${ }^{38,39}$ In addition, the immune response produced by larvae is very similar to that of mammals when facing fungal infection. ${ }^{38,39}$ Therefore, G. mellonella larvae models have been used increasingly in the study of the in vivo effects of drugs against fungal pathogens. $^{38-40}$ The results of the survival assay in Figure 1A proved that BEH plus FLC could significantly reduce the number of deaths of larvae compared with the other three groups. In addition, further experiments on the fungal burden and histological studies on larvae showed that the BEH plus FLC could not only reduce the amount of C. albicans in larvae but also significantly reduce the damage to larvae tissues as illustrated in Figures $1 \mathrm{~B}$ and 2. The in vivo data were consistent with the in vitro antifungal effect, indicating that BEH could indeed enhance the antifungal activities of FLC, and we hope the experiment could provide a data basis for further exploration of antifungal effects in mammalian models.

Moreover, we also explored the potential mechanisms of $\mathrm{BEH}$ alone or in combination with FLC against $C$. albicans. Calcium serves as the second messenger molecule, and it is important for the physiological process of cells in both fungi and mammals. ${ }^{6}$ Many studies have identified that calcium is closely associated with hyphal morphogenesis and acquired drug tolerance in C. albicans. ${ }^{41}$ Also, several agents could exert antifungal activities against $C$. albicans by affecting intracellular calcium homeostasis, such as beauvericin, verapamil, and silibinin. ${ }^{41-44}$ Our study found that $8 \mu \mathrm{g} / \mathrm{mL}$ $\mathrm{BEH}$ had an influence on $C$. albicans intracellular calcium homeostasis, resulting in a higher $\left[\mathrm{Ca}^{2+}\right]_{\mathrm{i}}$ compared with the control group, while the curve of BEH-FLC combination group was highly similar to $\mathrm{BEH}$ alone group. These findings indicated that $\mathrm{BEH}$ alone may exert an antifungal effect by interfering with intracellular calcium concentration, and the combined antifungal effect of BEH-FLC was not related to this mechanism.
Apoptosis is a physiological process leading to cell death in multicellular organisms, and it plays a vital role in the growth, development and maintenance of normal cells. ${ }^{45}$ Caspase activation is a sign of early cell apoptosis, it can participate in the degradation of specific proteins involved in apoptosis signal transduction, and then cause cell apoptosis. $^{46,47}$ Studies have proved that the functions of metacaspase in C. albicans are similar to that of caspase in mammals. ${ }^{48}$ In recent years, many researchers have proved that some antifungal drugs could exert an antifungal effect by activating the intracellular metacaspase in fungi, such as amphotericin B and echinocandins. ${ }^{4,49,50}$ Therefore, we used FITC-VAD-FMK In Situ Marker reagent to detect the activation of metacaspase in CA10 cells after intervention of different drugs. As shown in Figure 4, compared with the other three groups, we observed that the BEH-FLC combination group could produce obvious green fluorescence, which indicated that the intracellular metacaspase of CA10 was activated. Therefore, the synergistic antifungal effects of $\mathrm{BEH}$ combined with FLC may be related to the activation of intracellular metacaspase; however, the specific mechanism still needs to be further explored, which could provide new insights for overcoming fungal resistance.

In fungi and bacteria, up-regulation of the expression of efflux pump genes will increase the efflux of drugs and reduce the concentration of drugs, and it is one of the important reasons that drugs cannot effectively exert their antifungal effects. ${ }^{29,30,51-53}$ Therefore, increasing the accumulation of intracellular drugs by weakening the activity of the drug efflux pump is an effective strategy to overcome antibiotic resistance. Currently, it has been found that some compounds can indeed increase the sensitivity of antibiotics by inhibiting the activity of drug transporters in Staphylococcus aureus and Candida species. ${ }^{7,16,22,30,53}$ In this experiment, we determined the influence on FLC efflux of BEH through Rh6G efflux analysis. The results in Figure 5 had shown that the Rh6G efflux curves in the control group and BEH group were highly overlapped, and there was no significant difference. This suggested that BEH had no effect on FLC efflux, indicating that the synergistic effect of the BEH-FLC combination was unrelated to inhibit the efflux of FLC.

\section{Conclusion}

In conclusion, the present study demonstrated for the first time that $\mathrm{BEH}$ alone could exhibit antifungal activities against C. albicans. It could also enhance the antifungal activities of FLC against FLC resistant $C$. albicans both in vitro and in vivo. Mechanism studies have proved that the antifungal activity of 
$\mathrm{BEH}$ used alone or in combination with FLC was related to the increasement of intracellular calcium concentration and activation of metacaspase activity, and it was independent of FLC efflux in resistant $C$. albicans. Thus, it is promising for $\mathrm{BEH}$ to increase the sensitivity of antifungal drugs and overcome drug resistance. Further investigations are still needed for the application in the future.

\section{Ethical Approval}

The animal models used in this study are $G$. mellonella larvae, which do not require ethical approval.

\section{Acknowledgments}

We would like to thank the grants from the Beijing Health Alliance Charitable Foundation (WS630A).

\section{Disclosure}

The authors report a patent ZL202010782426.2 licensed to Shujuan Sun; Xueqi Chen; Chunyan Li; Haiying Yan; Hongyao Ren. The authors report no other conflicts of interest in this work.

\section{References}

1. Zavrel M, White TC. Medically important fungi respond to azole drugs: an update. Future Microbiol. 2015;10(8):1355-1373. doi:10. 2217/FMB.15.47

2. Ting SY, Ishola OA, Ahmed MA, et al. Metabolic adaptation via regulated enzyme degradation in the pathogenic yeast Candida albicans. J Mycol Med. 2017;27(1):98-108. doi:10.1016/j.mycmed.2016.12.002

3. Lopez-Ribot JL. Candida albicans biofilms: more than filamentation. Curr Biol. 2005;15(12):R453-5. doi:10.1016/j.cub.2005.06.020

4. Yapar N. Epidemiology and risk factors for invasive candidiasis. Ther Clin Risk Manag. 2014;10:95-105. doi:10.2147/TCRM.S40160

5. Anderson MZ, Saha A, Haseeb A, et al. A chromosome 4 trisomy contributes to increased fluconazole resistance in a clinical isolate of Candida albicans. Microbiology. 2017;163(6):856-865. doi:10.1099/ mic.0.000478

6. Li Y, Jiao P, Li Y, et al. The synergistic antifungal effect and potential mechanism of D-penicillamine combined with fluconazole against Candida albicans. Front Microbiol. 2019;10. doi:10.3389/fmicb.20 19.02853

7. Gong Y, Liu W, Huang X, et al. Antifungal activity and potential mechanism of N-butylphthalide alone and in combination with fluconazole against Candida albicans. Front Microbiol. 2019;10:1461. doi:10.3389/fmicb.2019.01461

8. Yoosefian M, Rahmanifar E, Etminan N. Nanocarrier for levodopa Parkinson therapeutic drug; comprehensive benserazide analysis. Artif Cells Nanomed Biotechnol. 2018;46(sup1):434-446. doi:10.10 80/21691401.2018.1430583

9. Li W, Zheng M, Wu S, et al. Benserazide, a dopadecarboxylase inhibitor, suppresses tumor growth by targeting hexokinase 2. J Exp Clin Cancer Res. 2017;36(1):58. doi:10.1186/s13046-017-0530-4

10. Laurian R, Dementhon K, Doumèche B, et al. Hexokinase and glucokinases are essential for fitness and virulence in the pathogenic yeast Candida albicans. Front Microbiol. 2019;10:327. doi:10.3389/ fmicb.2019.00327
11. Chen X, Zhang Z, Chen Z, et al. Potential antifungal targets based on glucose metabolism pathways of Candida albicans. Front Microbiol. 2020;11:296. doi:10.3389/fmicb.2020.00296

12. Kruszewska H, Zareba T, Tyski S. Examination of antimicrobial activity of selected non-antibiotic drugs. Acta Pol Pharm. 2004;61 (Suppl):18-21.

13. Lockhart SR, Bolden CB, Iqbal N, et al. Validation of 24-hour flucytosine MIC determination by comparison with 48-hour determination by the Clinical and Laboratory Standards Institute M27-A3 broth microdilution reference method. J Clin Microbiol. 2011;49 (12):4322-4325. doi:10.1128/JCM.05479-11

14. Liu X, Li T, Wang D, et al. Synergistic antifungal effect of fluconazole combined with licofelone against resistant Candida albicans. Front Microbiol. 2017;8:2101. doi:10.3389/fmicb.2017. 02101

15. Lu M, Yan H, Yu C, et al. Proton pump inhibitors act synergistically with fluconazole against resistant Candida albicans. Sci Rep. 2020;10 (1):498. doi:10.1038/s41598-019-57174-4

16. Li X, Zhao Y, Huang X, et al. Ambroxol hydrochloride combined with fluconazole reverses the resistance of Candida albicans to fluconazole. Front Cell Infect Microbiol. 2017;7:124. doi:10.3389/ fcimb.2017.00124

17. Odds FC. Synergy, antagonism, and what the chequerboard puts between them. J Antimicrob Chemother. 2003;52(1):1. doi:10.1093/ $\mathrm{jac} / \mathrm{dkg} 301$

18. Ramage G, Lopez-Ribot JL. Techniques for antifungal susceptibility testing of Candida albicans biofilms. Methods Mol Med. 2005;118:71-79. doi:10.1385/1-59259-943-5:071

19. Ramage G, Vande Walle K, Wickes BL, et al. Standardized method for in vitro antifungal susceptibility testing of Candida albicans biofilms. Antimicrob Agents Chemother. 2001;45(9):2475-2479. doi:10.1128/AAC.45.9.2475-2479.2001

20. Gago S, García-Rodas R, Cuesta I, et al. Candida parapsilosis, Candida orthopsilosis, and Candida metapsilosis virulence in the non-conventional host Galleria mellonella. Virulence. 2014;5 (2):278-285. doi:10.4161/viru.26973

21. Krezdorn J, Adams S, Coote PJ. A Galleria mellonella infection model reveals double and triple antibiotic combination therapies with enhanced efficacy versus a multidrug-resistant strain of Pseudomonas aeruginosa. J Med Microbiol. 2014;63(Pt 7):945-955. doi:10.1099/jmm.0.074245-0

22. Li Y, Yang J, Li X, et al. The effect of Ginkgolide B combined with fluconazole against drug-resistant Candida albicans based on common resistance mechanisms. Int J Antimicrob Agents. 2020;56 (2): 106030

23. Shi W, Chen Z, Chen X, et al. The combination of minocycline and fluconazole causes synergistic growth inhibition against Candida albicans: an in vitro interaction of antifungal and antibacterial agents. FEMS Yeast Res. 2010;10(7):885-893. doi:10.1111/j.15671364.2010.00664.x

24. Grynkiewicz G, Poenie M, Tsien RY. A new generation of $\mathrm{Ca} 2+$ indicators with greatly improved fluorescence properties. J Biol Chem. 1985;260(6):3440-3450. doi:10.1016/S0021-9258(19)83641-4

25. Chen X, Shi Y, Li Y, et al. Antifungal effects and potential mechanisms of lonidamine in combination with fluconazole against Candida albicans. Expert Rev Anti Infect Ther. 2021;19(1):109-115. doi:10. 1080/14787210.2020.1811684

26. Lu M, Yu C, Cui X, et al. Gentamicin synergises with azoles against drug-resistant Candida albicans. Int J Antimicrob Agents. 2018;51 (1):107-114. doi:10.1016/j.ijantimicag.2017.09.012

27. Malmquist JA, Rogan MR, McGillivray SM. Galleria mellonella as an infection model for Bacillus anthracis Sterne. Front Cell Infect Microbiol. 2019;9:360. doi:10.3389/fcimb.2019.00360

28. Gibreel TM, Upton M. Synthetic epidermicin NI01 can protect Galleria mellonella larvae from infection with Staphylococcus aureus. J Antimicrob Chemother. 2013;68(10):2269-2273. 
29. Bhattacharya S, Sobel JD, White TC. A combination fluorescence assay demonstrates increased efflux pump activity as a resistance mechanism in azole-resistant vaginal candida albicans isolates. Antimicrob Agents Chemother. 2016;60(10):5858-5866.

30. Monteiro KL, de Aquino TM, Mendonça Junior FJB. An update on Staphylococcus aureus NorA efflux pump inhibitors. Curr Top Med Chem. 2020;20(24):2168-2185. doi:10.2174/156802662066620070 4135837

31. Donadu MG, Peralta-Ruiz Y, Usai D, et al. Colombian essential oil of ruta graveolens against nosocomial antifungal resistant candida strains. J Fungi. 2021;7(5):383. doi:10.3390/jof7050383

32. Donadu M, Usai D, Marchetti M, et al. Antifungal activity of oils macerates of North Sardinia plants against Candida species isolated from clinical patients with candidiasis. Nat Prod Res. 2020;34 (22):3280-3284. doi:10.1080/14786419.2018.1557175

33. Pinna A, Donadu MG, Usai D, et al. In vitro antimicrobial activity of a new ophthalmic solution containing hexamidine diisethionate 0.05\%(keratosept). Cornea. 2020;39(11):1415-1418. doi:10.1097/ ICO.0000000000002375

34. Pinna A, Donadu MG, Usai D, et al. In vitro antimicrobial activity of a new ophthalmic solution containing povidone-iodine $0.6 \%\left(\right.$ IODIM $\left.^{\circledR}\right)$. Acta Ophthalmol. 2020;98(2):e178-e180. doi:10.1111/aos.14243

35. Li X, Yu C, Huang X, et al. Synergistic effects and mechanisms of budesonide in combination with fluconazole against resistant Candida albicans. PLoS One. 2016;11(12):e0168936. doi:10.1371/journal. pone. 0168936

36. Zhang M, Yan H, Lu M, et al. Antifungal activity of ribavirin used alone or in combination with fluconazole against Candida albicans is mediated by reduced virulence. Int J Antimicrob Agents. 2020;55 (1):105804. doi:10.1016/j.ijantimicag.2019.09.008

37. Kojic EM, Darouiche RO. Candida infections of medical devices. Clin Microbiol Rev. 2004;17(2):255-267. doi:10.1128/CMR.17.2.255-267. 2004

38. Brennan M, Thomas DY, Whiteway M, et al. Correlation between virulence of Candida albicans mutants in mice and Galleria mellonella larvae. FEMS Immunol Med Microbiol. 2002;34(2):153-157. doi:10.1111/j.1574-695X.2002.tb00617.x

39. Frenkel M, Mandelblat M, Alastruey-Izquierdo A, et al. Pathogenicity of Candida albicans isolates from bloodstream and mucosal candidiasis assessed in mice and Galleria mellonella. J Mycol Med. 2016;26(1):1-8. doi:10.1016/j.mycmed.2015.12.006

40. Mylonakis E, Moreno R, El Khoury JB, et al. Galleria mellonella as a model system to study Cryptococcus neoformans pathogenesis. Infect Immun. 2005;73(7):3842-3850. doi:10.1128/IAI.73.7.38423850.2005
41. Li Y, Sun L, Lu C, et al. Promising antifungal targets against Candida albicans based on ion homeostasis. Front Cell Infect Microbiol. 2018;8:286. doi:10.3389/fcimb.2018.00286

42. Tong Y, Liu M, Zhang Y, et al. Beauvericin counteracted multi-drug resistant Candida albicans by blocking ABC transporters. Synth Syst Biotechnol. 2016;1(3):158-168. doi:10.1016/j.synbio.2016.10.001

43. Yu Q, Ding X, Zhang B, et al. Inhibitory effect of verapamil on Candida albicans hyphal development, adhesion and gastrointestinal colonization. FEMS Yeast Res. 2014;14(4):633-641. doi:10.1111/ 1567-1364.12150

44. Yun DG, Lee DG. Silibinin triggers yeast apoptosis related to mitochondrial $\mathrm{Ca}(2+)$ influx in Candida albicans. Int J Biochem Cell Biol. 2016;80:1-9. doi:10.1016/j.biocel.2016.09.008

45. Shirazi F, Kontoyiannis DP. Micafungin triggers caspase-dependent apoptosis in Candida albicans and Candida parapsilosis biofilms, including caspofungin non-susceptible isolates. Virulence. 2015;6 (4):385-394. doi:10.1080/21505594.2015.1027479

46. Shirtliff ME, Krom BP, Meijering RAM, et al. Farnesol-Induced Apoptosis in Candida albicans. Antimicrob Agents Chemother. 2009;53(6):2392-2401. doi:10.1128/AAC.01551-08

47. Hengartner MO. Apoptosis - DNA destroyers. Nature. 2001;412 (6842):27-29. doi:10.1038/35083663

48. Yun J, Lee DG. Cecropin A-induced apoptosis is regulated by ion balance and glutathione antioxidant system in Candida albicans. IUBMB Life. 2016;68(8):652-662. doi:10.1002/iub.1527

49. Hao B, Cheng S, Clancy CJ, et al. Caspofungin kills candida albicans by causing both cellular apoptosis and necrosis. Antimicrob Agents Chemother. 2013;57(1):326-332. doi:10.1128/AAC.01366-12

50. Phillips AJ, Sudbery I, Ramsdale M. Apoptosis induced by environmental stresses and amphotericin B in Candida albicans. Proc Natl Acad Sci U S A. 2003;100(24):14327-14332. doi:10.1073/ pnas. 2332326100

51. Mishra NN, Prasad T, Sharma N, et al. Pathogenicity and drug resistance in Candida albicans and other yeast species. A review. Acta Microbiol Immunol Hung. 2007;54(3):201-235. doi:10.1556/ amicr.54.2007.3.1

52. Viet-Khoa T-N, Prasad R, Falson P, et al. Modulators of the efflux pump Cdr1p of Candida albicans: mechanisms of action and chemical features. Curr Med Chem. 2017;24(30):3242-3253. doi:10.2174/ 0929867324666170523102244

53. Usai D, Donadu M, Bua A, et al. Enhancement of antimicrobial activity of pump inhibitors associating drugs. $J$ Infect Devel Countries. 2019;13(02):162-164. doi:10.3855/jidc.11102

\section{Publish your work in this journal}

Drug Design, Development and Therapy is an international, peerreviewed open-access journal that spans the spectrum of drug design and development through to clinical applications. Clinical outcomes, patient safety, and programs for the development and effective, safe, and sustained use of medicines are a feature of the journal, which has also been accepted for indexing on PubMed Central. The manuscript management system is completely online and includes a very quick and fair peer-review system, which is all easy to use. Visit http://www. dovepress.com/testimonials.php to read real quotes from published authors. 